\title{
Effect of Polyvinyl Pyrolidone on Morphology and Performance of Cellulose Acetate Based Dialysis Membrane
}

\author{
Hizba Waheed \\ School of Chemicals and Materials Engineering \\ National University of Sciences and Technology (NUST) \\ Islamabad, Pakistan \\ hizbawaheed@scme.nust.edu.pk
}

\author{
Arshad Hussain \\ School of Chemical and Materials Engineering \\ National University of Sciences and Technology (NUST) \\ Islamabad, Pakistan \\ arshad.hussain@scme.nust.edu.pk
}

\begin{abstract}
Polyvinyl pyrolidone (PVP) was added as filler in cellulose acetate (CA) to produce mixed matrix membrane (MMM) for hemodialysis operation. Phase separation induced by diffusion (DIPS) was used for fabrication of mixed matrix CA/PVP flat sheet membranes. The effect of adding PVP was investigated on the morphology and permeation efficiencies of CA membranes. The surface arrangement of polymer and additives in pure and blended membrane was studied by FTIR, contact angle and SEM. Results revealed homogenous and significant mixing of PVP content into pure CA matrix. Performance efficiency of blended membranes was investigated by means of pure water flux (PWF), urea clearance and \% rejection of bovine serum albumin (BSA). The observable decrease of contact angle from $83^{\circ}$ to $69^{\circ}$ in CA/PVP MMM membranes of varying composition effectively revealed enhancement in hydrophilicity of MMM membrane surface. For protein rejection, all CA/PVP membranes rejected $>90 \%$ of $\mathrm{BSA}$ relative to $25 \%$ for pure $\mathrm{CA}$ membrane. Furthermore, urea clearance behavior for CA/PVP membranes was $62.4 \%$ in comparison to $52 \%$ for pure CA membrane. The incorporation PVP i.e 1\% by weight (Mpvp1) significantly improved the hydrophilicity, PWF, BSA rejection and urea clearance percentages of modified CA membrane for dialysis application.
\end{abstract}

Keywords-cellulose acetate; dialysis; membrane; poly vinyl pyrolidone

\section{INTRODUCTION}

Kidney problems either acute or chronic are one the major mortality causes. Diseases related to kidneys have increased tremendously, main reasons being diabetes and hypertension which are common nowadays [1-4]. The increase in the amount of uremic toxics beyond the normal range within blood, effect adversely and is called uremic syndrome. To prolong life, kidney patients undergo dialysis which is an expensive procedure and costs more than 1 billion US\$ annually [5]. Hemodialysis is an important medical procedure for patients suffering from renal ailments. This process includes purification of blood from patient's body using hemodialysis equipment (artificial kidney) to remove uremic wastes and returning the filtered blood back to person's body [6]. The important part of hemodialysis apparatus is a membrane, which function is to separate toxic wastes produced as a result of metabolic activities and excess water from the patient through transport via diffusive and convective phenomena. During the process, essential protein loss is controlled or prevented via pore size exclusion of the membrane [7]. Keeping importance of hemodialysis in view, progress is made in research field of high-performance membrane which will lead to enhance quality of treatment and increase patient's life.

Different polymers used for dialysis membrane preparation other than cellulose-based materials include polysulfone (PS), polyacrilonitrile (PAN), polymethyl methacrylate (PMMA), polyamide (PA), and polyvinylalcohol (PVA) [8-12]. Many additives have been used to enhance dialysis performance of prepared membranes. Among them are polyethylene glycol (PEG), monosodium glutamate, polyethylene imine (PEI) etc. In the present study, CA was chosen as a basic polymer for the fabrication of flat sheet dialysis membranes. Phase inversion technique is commonly used for the preparation of asymmetric flat sheet membranes. Casting solutions were made from CA in acetic acid as solvent and pure water as non-solvent agent. Choice of cellulose was made on the bases of its easy availability and low cost. The only drawback for CA is its nonbiocompatibility. In order to overcome this problem, a biocompatible additive with good hydrophilicity was added to CA so that the synthesized membranes can have enhanced dialysis efficiency. PVP is hydrophilic additive which enhances the hydrophilic behavior of CA membranes. It is a watersoluble polymer which increases the porosity of membranes [13], and has good blood compatibility [14]. Keeping that in mind, the casting solution was later modified using polyvinyl pyrolidone (PVP) in various weight percentages.

Surface morphology, functional group identification, pure water flux, bovine serum albumin rejection percentage and urea clearance values of CA/PVP were inspected and were compared with pure CA flat sheet membranes. The purpose of this research was to synthesize a new kind of CA/PVP membranes with high BSA rejection and better urea clearance for dialysis application. 


\section{MATERIALS AND METHODS}

\section{A. Materials}

Asymmetric polymeric membranes were synthesized for dialysis application. CA polymer with average mol. wt of 30,000 Daltons having $39.8 \%$ degree of acetylation, $99.7 \%$ pure acetic acid, $98 \%$ pure urea with mol. wt 60.06 , Bovine serum with mol. wt of 66,000 Dalton and PEG with mol.wt 10,000 and 35,000 were obtained from Sigma Aldrich. PVP with average mol. wt. 30,000 was purchased from Fluka.

\section{B. Membrane Synthesis}

Several casting solutions of CA/PVP/Acetic acid blend were prepared having constant CA weight $\%$ of 18.5 . Table I shows the compositions of casting solutions prepared during this work. The casting solutions were agitated constantly to confirm complete mixing of added ingredients including polymer, solvent and additive. When the casting solution showed complete dissolution of polymer and PVP, it was degassed for 2 hours using ultrasonic bath to remove trapped air bubbles. Complete mixing of components is indicated by formation of clear solution. To slow down the aging process the casting solutions were kept in dark after degassing. The casting solution was poured on a glass plate and was spread with a doctor blade having wet membrane thickness of $200 \mu \mathrm{m}$. The glass plate was instantly submerged in distilled water trough where phase separation took place [15]. During phase separation, exchange on solvent (acetic acid) and non-solvent (water) occurred. The prepared flat sheet membrane was then shifted to another trough containing fresh water and was washed to get rid of any excess solvent if present. Washing was carried out for 3-4 times. Finally, the casted membrane was kept in distilled water for 24 hours before final testing.

TABLE I. COMPOSITION OF MEMBRANES

\begin{tabular}{|c|c|c|c|}
\hline Membrane & CA wt. \% & PVP wt. \% & Acetic acid wt. \% \\
\hline Mpvp0 & 18.5 & - & 80.5 \\
\hline Mpvp1 & 18.5 & 1.00 & 81.5 \\
\hline Mpvp3 & 18.5 & 3.00 & 78.5 \\
\hline Mpvp5 & 18.5 & 5.00 & 76.5 \\
\hline Mpvp7 & 18.5 & 7.00 & 74.5 \\
\hline
\end{tabular}

It was observed that when we increased the PVP concentration beyond 5\% the membranes formed were having visible pores and the casting was not possible as it was for the rest of the membranes. The visible physical difference in membranes with concentration less than $5 \%$ and beyond $5 \%$ is shown in Figure 1.

\section{MEMBRANE TESTING (WET)}

\section{A. Pure Water Flux}

Dead end filtration setup was used to calculate pure water permeation flux (PWP) for dialysis membrane. The filtration cell was having $250 \mathrm{ml}$ capacity for feed solution and an active area of $12.57 \mathrm{~cm}^{2}$. Permeation experiments were operated under 2.0-3.0bar pressure. All experiments were carried out at ambient temperature at $25 \pm 0.5^{\circ} \mathrm{C}$ as shown in Figure 2. The fabricated membrane was positioned in test cell and was tested for water flux. Experiments were repeated several times to avoid data ambiguity. The PWP flux was measured using (1):

$$
\text { Flux }(J)=Q / \Delta t \times A
$$

In (1), $J$ represents the permeation flux (Lm-2h-1) for pure water, $Q$ is the volume of permeated solution, $A$ shows the active area of membrane and $\Delta t$ is the permeation time (h) [16]. A similar method was used to analyze molecular weight cutoff for the fabricated membranes. Solutions of BSA (average mol wt 66000) and PEG with different molecular weights (PEG 10,000 and PEG 35,000) were used. All solutions were made having concentration of $1 \mathrm{~g} / \mathrm{L}$. The concentrations of PEG and BSA solutions were found using Dragondorff reagent [17, 18] by UV-spectrophotometer.
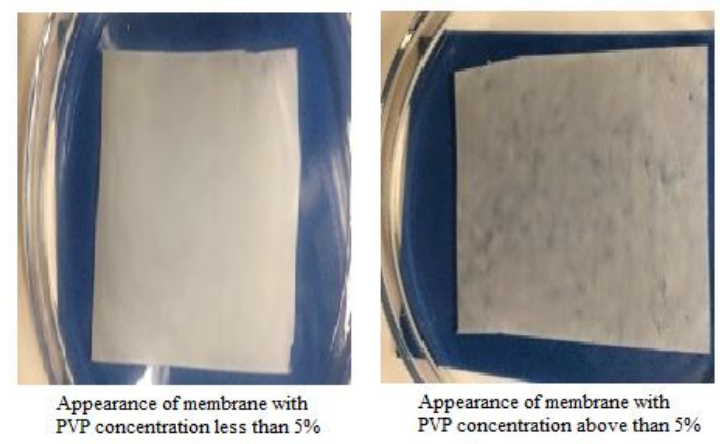

Fig. 1. Difference in apearance of membranes.

\section{B. Hydrophilicity Measurement}

Contact angle and water uptake values were used to measure the CA/PVP membrane hydrophilicity. Sessile drop method was used to measure contact angle at a Tantec contact angle meter $[19,20]$. Single drop of deionized water was released on a dried membrane flat surface using a microsyringe. The tests were made under saturated water vapor atmosphere and the contact angle was measured after 10s. Minimum 8 contact angle readings were made to get an average value and all readings were taken at room temperature. To calculate water uptake/absorption, the membrane sample $(2 \mathrm{~cm} \times 2 \mathrm{~cm})$ was cut and desiccated in an oven at $60^{\circ} \mathrm{C}$ for $12 \mathrm{~h}$ prior to testing. The dried piece was weighed $\left(M_{d r y}\right)$. The preweighed membrane sample was then waterlogged at room temperature for the next $24 \mathrm{~h}$. The waterlogged membrane was taken out and dapped with tissue paper to remove water. The membrane piece was weighed again $\left(M_{w e t}\right)$. All measurements were made at $25 \pm 0.5^{\circ} \mathrm{C}$. The water uptake was determined using (2) where $M_{w e t}$ and $M_{d r y}$ represent the wet and dry membrane weights:

$$
\text { Water take up }=\frac{M w e t-M d r y}{M d r y} \times 100
$$

\section{MEMBRANE PERFORMANCE}

Membrane performance tests were based on the extent of removing uremic toxins and rejecting bovine serum albumin from feed solution. Urea clearance and BSA rejection was measured separately using dialysis setup and dead-end filtration method. For urea clearance, the experiments were performed in a dialyzer with one compartment (donor) containing $50 \mathrm{ml}$ feed solution and the other (receiver) filled with $2 \mathrm{~L}$ distilled water as shown in Figure 2. 


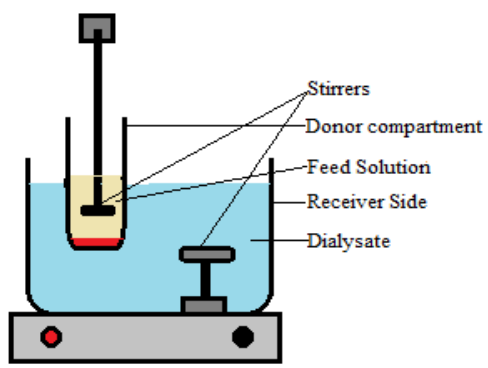

Fig. 2. Schematic presentation of dialysis setup.

Two similar stirrers were fitted in the compartments. The membrane to be tested was clamped between the two chambers. The concentrations of urea and BSA were kept similar to their concentrations in human blood. For urea clearance, experiments were started by bringing the feed solution in contact with the testing membrane. The concentration gradients of solute (urea or BSA) in two sides of the dialysis membrane act as the driving forces for the transport of urea and BSA from the feed side to the distilled water side. The concentration of urea was measured every $30 \mathrm{~min}$ from the start of the experiments and the experiment was performed continuously for $210 \mathrm{~min}$. Urea concentrations were tested using total organic carbon analyzer methods and UVspectrophotometric method was applied for measuring BSA concentration. The membrane performance, i.e. urea clearance and BSA rejection was calculated from (3) where $C_{i}$ and $C_{f}$ represent initial and final concentrations respectively.

Urea clearance $\%=\frac{C i-C f}{C i} X 100$

BSA rejection \% was estimated using dead end filtration cell (Figure 3). A feed solution having concentration $1 \mathrm{mg} / \mathrm{ml}$ BSA was prepared. The active membrane area was $12.57 \mathrm{~cm}^{2}$. The quantity of BSA in feed and permeate was measured by observing its absorbance with a spectrophotometer (Shidmazu UV mini 1240) operated at a wavelength of $278 \mathrm{~nm}$. The experiments were carried out at room temperature at 2-3bar pressure by employing (4) where $C_{p}, C_{r}$ stand for permeate and retentate concentrations respectively [21].

$$
\text { Percent Rejection }=\left(1-\frac{C p}{C r}\right) \times 100
$$

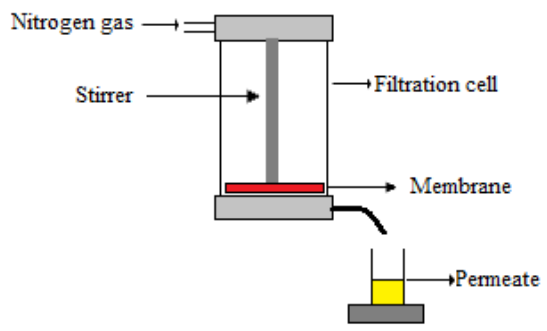

Fig. 3. Dead end filtration setup.

\section{MEMBRANE CHARACTERIZATION}

The cross-section and surface morphologies of casted membranes were analyzed using a JSM 6409A scanning electron microscope. The membranes were first frozen in liquid nitrogen and later were broken and spluttered with gold before undergoing SEM analysis. FTIR Spectrum 100 PerkinElmer, MID IR was used to carry out FTIR analysis of prepared membranes. The membrane pieces were cut and placed in a pallet holder one after the other. The holder was then fixed in an instrument. Wave numbers from 450 to $4000 \mathrm{~cm}^{-1}$ were used to record the spectra in transmission mode. This procedure was repeated for every sample separately.

\section{RESULTS AND DISCUSSION}

\section{A. Effect of PVP Addition on Morphology and Performance of CA Membranes}

SEM investigation was made to study the effects of adding PVP to CA matrix. Figure 4 shows the micrographic view of surface morphology and cross-sectional arrangements of polymer and additive in all prepared membranes. As revealed in the surface morphology, addition of PVP to CA matrix leads to the generation of asymmetric membranes with compact top layer and sub-layer with finger like porous structures all over. From these images, it is seen that keeping bath temperature constant at $25^{\circ} \mathrm{C}$ and rising PVP concentration from 0 to $3 \mathrm{wt} . \%$ caused development of macro voids and porous structure with good water flux. However, further increment of PVP concentration beyond $4 \mathrm{wt} . \%$ resulted in formation of large visible pores making membranes not functioning for filtration, purification and dialysis operations. It was observed that the existence of hydrophilic additive PVP resulted in immediate demixing in the coagulation bath and generates macro voids in membrane structure till the concentration was below 3wt.\% $[22,23]$. Whereas due to low affinity between PVP and CA polymer, large pores were formed when wt.\% was increased beyond 4wt.\% as shown in Figures 1 and 4. Conclusively, it is said that addition of a hydrophilic additive PVP, to the CA polymeric solution induces a dual effect on the membrane morphology. The membrane structure attained is subjected to the dominance of instantaneous or late demixing. These both phenomena are occurring because of the presence of the PVP casting matrix. Conferring to Figure 4, adding PVP from 0 to 3 wt. $\%$ causes development of pores and voids in the membranes. However, increasing PVP concentration beyond $3 \%$ in CA matrix, results in formation of larger finger like projections and highly porous structures. Therefore, it is concluded that little addition of PVP, i.e up to $3 \mathrm{wt} \%$, promotes immediate demixing, whereas further increment of PVP, beyond 3 wt. \%, results in formation of membranes with large and visible pores.

\section{B. FTIR Analysis of CA/PVP Blended Membranes}

Addition of PVP to CA matrix resulted in the broadening and shifting of bands and peaks in FTIR spectrum (Figure 5). FTIR spectrum of CA/PVP blended membranes presented prominent carbonyl group $(-\mathrm{C}=\mathrm{O})$ stretching vibration of at $\sim 1734 \mathrm{~cm}^{-1}$ and stretching vibration of $-\mathrm{CH}$ group at $\sim 2960 \mathrm{~cm}^{-1}$, which are defining peaks of CA [24, 25]. Mpvp1, Mpvp2 and Mpvp3 also showed stretching vibration of ( $\mathrm{C}=\mathrm{O}-\mathrm{N}$ ) amide carbonyl group at $\sim 1656 \mathrm{~cm}^{-1}$, bending vibration of $-\mathrm{CH} 2-$ group at $\sim 1498 \mathrm{~cm}^{-1}$. C-N group also exhibited stretching vibration of tertiary at $\sim 1295 \mathrm{~cm}^{-1}[26,27]$. 
It is visible from the spectrum that as wt. \% concentration increases, the peaks are becoming sharper. Mpvp5 shows the same trend as Mpvp1 and Mpvp3 but the values were small so not as prominent as in the case of the other two membranes. So, the presence of PVP in CA matrix is confirmed by the bands and peaks shown by Mpvp1, Mpvp3 and Mpvp5.

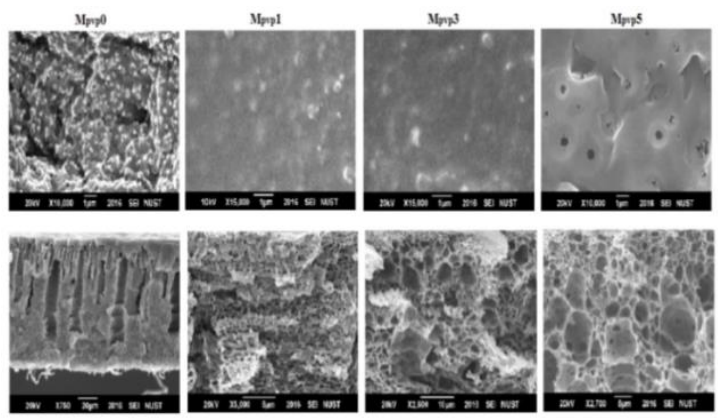

Fig. 4. SEM images showing the surface.

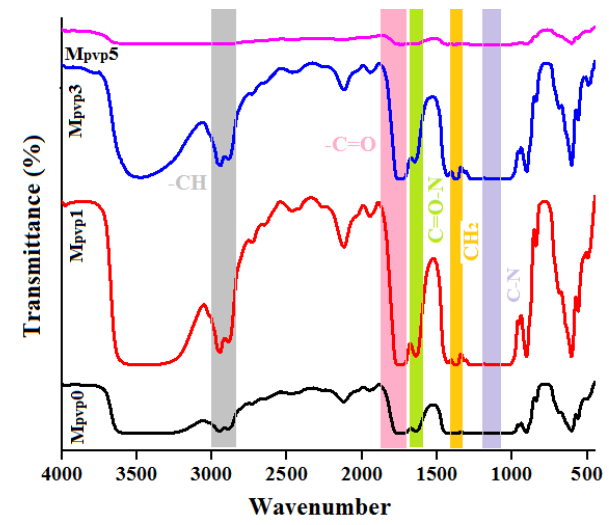

Fig. 5. FTIR spectrum of CA and CA/PVP blended membranes.

\section{Effect of PVP Concentration on Hydrophilicity and Water Uptake}

Water absorption or water uptake capacities and contact angle measurements are commonly used to estimate the extent of hydrophilic or hydrophobic nature of the membrane. If a membrane has low contact angle and possesses high-water absorption then it is high hydrophilic. Contact angle values and water absorption measurements for $\mathrm{CA}$ and modified CA membranes are shown in Figure 6. It is seen that water absorption of the PVP membrane increases and increasing PVP concentration up to $5 \%$ decreased the contact angle. These results indicated that the assimilation of PVP can increase the hydrophilicity for CA membrane obviously.

\section{BSA Rejection \% Study of CA/PVP Blended Membranes}

Loss of albumin $(\approx 67 \mathrm{kDa})$ occurs while renal patient undergoes dialysis treatment. For good dialysis operation, albumin loss should be avoided during operation. Figure 7 signifies the \% rejection of all pure $\mathrm{CA}$ and $\mathrm{CA} / \mathrm{PVP}$ blend membranes. All membranes except pure CA have BSA rejection above $90 \%$ while membranes Mpvp1, Mpvp3 and Mpvp5 have rejection of $96.7 \%, 97.8 \%$ and $99.2 \%$ respectively which is relatively attractive for commercial dialysis membranes to avoid albumin loss [28]. Membrane Mpvp1 with $96.7 \%$ rejection gives optimum properties for example urea clearance, contact angle, water uptake and water flux concerning dialysis.

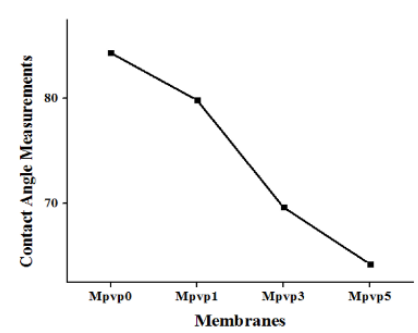

(a)

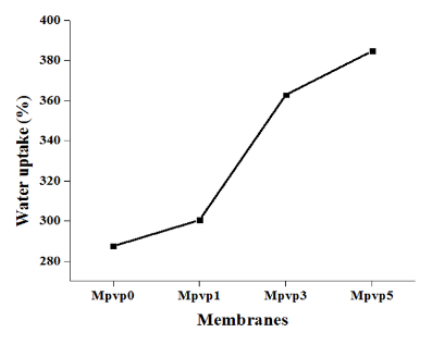

(b)
Fig. 6. (a) Contact angle and (b) water uptake of CA/PVP blended membranes.

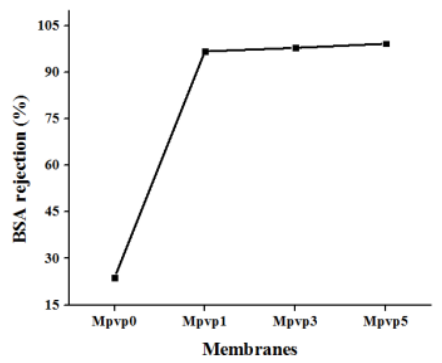

Fig. 7. BSA Rejection of CA/PVP blended membranes.

\section{E. Effect of PVP Concentration and Evaporation Time on Pure Water Flux}

The desired membranes were prepared with different evaporation times of $0,10,20$ and $30 \mathrm{sec}$. The prepared membranes were tested for pure water flux calculation using dead end filtration arrangement and it was found that evaporation time has a visible impact on CA/PVP blended membrane's performance. Increasing evaporation time affects inner structure and compaction of polymeric and additive particles within the membrane structure. The greater the evaporation time, the more suitable is the pore formation and uniform is the distribution of pores on membrane surface. From Figure 8 , it is visible that the best membranes were casted when given 30 s evaporation time to the casted membrane. These membranes gave optimum values of flux.

\section{F. Urea Clearance Study of CA/PVP Blended Membranes}

Urea clearance study is one of the major studies made to investigate the efficiency of hemodialysis membranes. Removal of waste including urea, excess water etc. is essential to maintain balance in patient's blood. For this purpose, prepared membranes were examined using the experimental arrangement shown in Figure 2. A fine dialysis membrane should have urea clearance value of at least $60 \%$. Figure 9 demonstrates the \% urea reduction of different CA/PVP blended membranes. Sample Mpvp1 exhibited urea reduction of $62 \%$ in comparison to pure CA membrane with $52.1 \%$, which is better than considerations made for a good commercial dialysis membrane [29]. The percentage of urea clearance of Mpvp0, Mpvp3 and Mpvp5 is shown in Figure 9. 


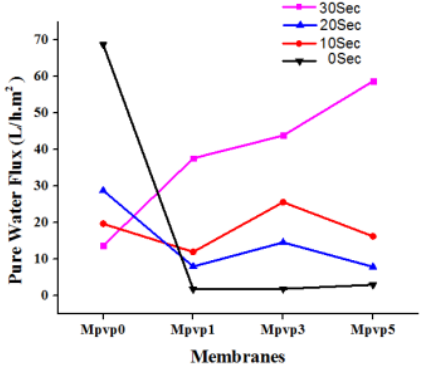

Fig. 8. Graphical presentation of effect of evaporation time on pure water flux.

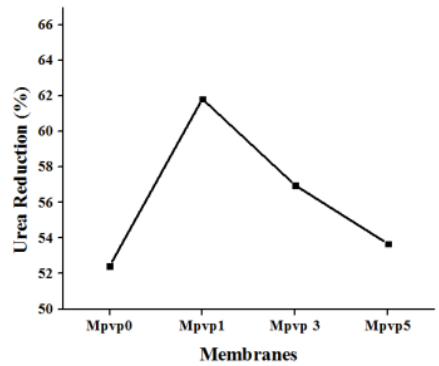

Fig. 9. Urea clearance of CA/PVP blended membranes.

\section{CONCLUSION}

During this research, PVP was used as additive for the manufacture of CA flat sheet membranes using phase inversion method. CA/PVP membranes showed compact structure with porous layer having macro voids when viewed in cross section. SEM and AFM characterization confirmed the uniform distribution of pores resulting in roughness at surface. FTIR studies revealed the effective bonding between PVP and CA. The hydrophilic nature was also studied by under taking contact angle and water uptake experiments. All these characterizations showed that hydrophilicity increased with addition of PVP to CA matrix. The performance testing showed that the efficiency of CA/PVP blended membranes was enhanced in terms of PWP flux, BSA rejection \% and urea clearance values. This study revealed that PVP is an appropriate additive to enhance uremic waste clearance. Conclusively, the CA/PVP blended membranes can be used as a capable solution for hemodialysis treatment after some appropriate conditional changes.

\section{ACKNOWLEDGMENT}

This work was supported by the School of Chemical and Materials Engineering, National University of Science and Technology, Islamabad, Pakistan and Chemical Engineering Department of University of Waterloo, Ontario, Canada.

\section{REFERENCES}

[1] S. Farrukh, A. Hussain, N. Iqbal, "Fabrication and characterization of microfiltration blended membranes", Desalination and Water Treatment, Vol. 52, No. 10-12, pp. 1833-1840, 2013

[2] S. Farrukh, F. T. Minhas, A. Hussain, S. Memon, M. I. Bhanger, M. Mujahid, "Preparation, Characterization and applicability of Novel Calix[4]arene based cellulose acetate membrane in gas permeation", Journal of Applied Polymer Science, Vol. 131, No. 6, 2014
[3] A. Hussain, S. Farrukh, F. T. Minhas, "Two-stage membrane system for post-combustion CO2 capture application", Energy \& Fuels,Vol. 29, No. 10, pp. 6664-6669, 2015

[4] S. S. Hayek, S. Sever, Y. A. Ko, H. Trachtman, M. Awad, S. Wadhwani, M. M. Altintas, C. Wei, A. L. Hotton, A. L. French, L. S. Sperling, S. Lerakis, A. A. Quyyumi, J. Reiser, "Soluble urokinase receptor and chronic kidney disease", The New England Journal of Medicine, Vol. 373, pp. 1916-1925, 2015

[5] C. C. Striemer, T. R. Gaborski, J. L. McGrath, P. M. Fauchet, "Chargeand size-based separation of macromolecules using ultrathin silicon membranes", Nature, Vol. 445, No. 7129, pp. 749-753, 2007

[6] C. Legallais, G. Catapano, B. V. Harten, U. Baurmeister, "A theoretical model to predict the in vitro performance of hemodiafilters", Journal of Membrane Science, Vol. 168, No. 1-2, pp. 3-15, 2000

[7] A. Zelman, D. Gisser, G. Strait, V. Bastidas, R. Stephen, C. Kablitz, J. Harrow, B. Deeter, W. J. Kolff, "Pressure control of the ultrafiltration rate during hemodialysis with high-flux dialyzers and the time dependence of membrane transport parameters", Synthetic Membranes: Volume II, pp. 61-74, American Chemical Society, 1981

[8] T. Kanamori, K. Sakai, T. Awaka, M. Fukuda, "An improvement on the method of determining the solute permeability of hollow-fiber dialysis membranes photometrically using optical fibers and comparison of the method with ordinary techniques", Journal of Membrane Science, Vol. 88, No. 2-3, pp. 159-165, 1994

[9] J. Ethkve, P. Dkjardin, M. Boissere, "Influence of $\mathrm{pH}$ on the adsorption of lysozyme on a sulfonated membrane with and without poly(ethyleneimine)", Desalination, Vol. 146, No. 1-3, pp. 111-113, 2002

[10] H. Kataoka, T. Kunitomo, T. Kobayashi, Hemodialysis Method and Membrane, US Patent 4925534, 1990

[11] A. Mochizuki, Y. Seita, T. Nakashima, F. Endo, S. Yamashita, "Polyether-Segmented Nylon Hemodialysis Membrane. V. Evaluation of Blood Compatibility of Polyether-Segmented Nylons", Journal of Applied Polymer Science,Vol. 67, No. 7, pp. 1253-1257, 1998

[12] J. Barzin, J. S. Madaeni, H. Mirzadeh, "Effect of Preparation Conditions on Morphology and Performance of Hemodialysis Membranes Prepared from Polyether Sulphone and Polyvinylpyrrolidone", Iranian Polymer Journal, Vol. 14, No. 4, pp. 353-360, 2005

[13] A. Higuchia, K. Shiranoa, M. Harashimaa, B. O. Yoona, M. Haraa, M. Hattorib, K. Imamurac, "Chemically modified polysulfone hollow fibers with vinylpyrrolidone having improved blood compatibility", Biomaterials, Vol. 23, No. 13, pp. 2659-2666, 2002

[14] S. Mei, C. Xiao, X. Hu, "Preparation of Porous PVC Membrane via a Phase Inversion Method from PVC/DMAc/Water/Additives", Journal of Applied Polymer Science, Vol. 120, No. 1, pp. 557-562, 2011

[15] M. Sivakumar, A. K. Mohana Sundaram, D. Mohan, K. Balu, R. Rangrajan, "Modification of Cellulose Acetate: Its Characterization and Application as an Ultrafiltration Membrane", Journal of Applied Polymer Science, Vol. 67, No. 11, pp. 1939-1946, 1998

[16] M. Irfan, A. Idris, N. M. Yusof, N. F. M. Khairuddin, H. Akhma, "Surface modification and performance enhancement of nano-hybrid $\mathrm{f}$ MWCNT/PVP90/PES hemodialysis membranes", Journal of Membrane Science, Vol. 467, pp. 73-84, 2014

[17] Z. Jia, C. Tian, "Quantitative determination of polyethylene glycol with modified Dragendorff reagent method", Desalination, Vol. 247, No. 1-3, pp. 423-429, 2009

[18] J. H. Kim, K. H. Lee, "Effect of PEG additive on membrane formation by phase inversion", Journal of Membrane Science, Vol. 138, No. 2, pp. 153-163, 1998

[19] A. Idris, L. K. Yet, "The effect of different molecular weight PEG additives on cellulose acetate asymmetric dialysis membrane performance", Journal of Membrane Science, Vol. 280, No. 1-2, pp. 920-927, 2006

[20] C. M. Kee, A. Idris, "Permeability performance of different molecular weight cellulose acetate hemodialysis membrane", Separation and Purification Technology, Vol. 75, No. 2, pp. 102-113, 2010

[21] J. J. Qin, M. H. Oo, Y. M. Cao, L. S. Lee, "Development of a LCST membrane forming system for cellulose acetate ultrafiltration hollow 
fiber", Separation and Purification Technolog, Vol. 42, No. 3, pp. 291295,2005

[22] I. C. Kim, K. H. Lee, "Effect of poly(ethylene glycol) 200 on the formation of a polyetherimide asymmetric membrane and its performance in aqueous solvent mixture permeation", Journal of Membrane Science, Vol. 230, No. 1-2, pp. 183-188, 2004

[23] M. M. Castillo-Ortega, A. Najera-Luna, D. E. Rodriguez-Felix, J. E. Encinas, F. Rodriguez-Felix, J. Romero, P. J. Herrera-Franco, "Preparation, characterization and release of amoxicillin from cellulose acetate and poly(vinyl pyrrolidone) coaxial electrospun fibrous membrane", Materials Science and Engineering: C, Vol. 31, No. 8, pp. 1772-1778, 2011

[24] Z. Cai , X. Song, Q. Zhang, T. Zhai, "Electrospun Polyindole Nanofibers as a Nano-adsorbent for Heavy Metal Ions Adsorption for Wastewater Treatment", Fibers and Polymers, Vol. 18, No. 3, pp. 502-513, 2017

[25] S. Ramesh, R. Shanti, E. Morris, "Characterization of conducting cellulose acetate based polymer electrolytes doped with "green" ionic mixture", Carbohydrate Polymers,Vol. 91, No. 1, pp. 14-21, 2013

[26] J. Bai, Y. Li, C. Zhang, X. Liang, Q. Yang, "Preparing $\mathrm{AgBr}$ nanoparticles in poly(vinyl pyrrolidone) (PVP) nanofibers", Colloids and Surfaces A: Physicochemical and Engineering Aspects, Vol. 329, No. 3, pp. 165-168, 2008

[27] M. Gao, L. Sun, Z. Wang, Y. Zhao, "Controlled synthesis of Ag nanoparticles with different morphologies and their antibacterial properties", Materials Science and Engineering: C, Vol. 33, No. 1, pp. 397-404, 2013

[28] Y. Iwasaki, H. Yamato, T. N. K. A. Fujieda, U. Uchida, A. H. M. Motojima, M. Fukagawa, "Uremic toxin and bone metabolism", Journal of bone and mineral metabolism,Vol. 24, No. 2, pp. 172-175, 2006

[29] G. Eknoyan, G. J. Beck, A. K. Cheung, J. T. Daugirdas, T. Greene, J. W. Kusek, M. Allon, J. Bailey, J. A. Dalmez, T. A. Depner, J. T. Dwyer, A. S. Levey, N. W. Levin, E. Milford, D. B. Ornt, M. V. Rocco, G. Schulman, S. J. Schwan, B. P. Teehan, R. Toto, "Effect of dialysis dose and membrane flux in maintenance hemodialysis", The New England Journal of Medicine, Vol. 347, No. 25, pp. 2010-2018, 2002 